\title{
Glucocorticoids Increase Excitotoxic Injury and Inflammation in the Hippocampus of Adult Male Rats
}

\author{
Shawn F. Sorrells ${ }^{\mathrm{a}}$ Carolina D. Munhoz $^{\mathrm{d}}$ Nathan C. Manley ${ }^{\mathrm{a}, \mathrm{b}}$ Sandra Yen ${ }^{\mathrm{a}}$ \\ Robert M. Sapolsky ${ }^{\mathrm{a}-\mathrm{c}}$ \\ Departments of a Biology, ${ }^{b}$ Neurosurgery and cNeurology and Neurological Sciences, Stanford University, Stanford, \\ Calif., USA; ${ }^{d}$ Department of Pharmacology, Institute of Biomedical Science, University of São Paulo, São Paulo, Brazil
}

\section{Key Words}

Inflammation · Excitotoxicity $\cdot$ Stress $\cdot$ Minocycline $\cdot N F-K B$

\begin{abstract}
Background/Aims: Stress exacerbates neuron loss in many CNS injuries via the actions of adrenal glucocorticoid (GC) hormones. For some injuries, this GC endangerment of neurons is accompanied by greater immune cell activation in the CNS, a surprising outcome given the potent immunosuppressive properties of GCs. Methods: To determine whether the effects of GCs on inflammation contribute to neuron death or result from it, we tested whether nonsteroidal antiinflammatory drugs could protect neurons from GCs during kainic acid excitotoxicity in adrenalectomized male rats. We next measured GC effects on (1) chemokine production (CCL2 and CINC-1), (2) signals that suppress immune activation (CX3CL1, CD22, CD200, and TGF- $\beta$ ), and (3) NF-kB activity. Results: Concurrent treatment with minocycline, but not indomethacin, prevented GC endangerment. GCs did not substantially affect CCL2, CINC-1, or baseline NF-KB activity, but they did suppress CX3CL1, CX3CR1, and CD22 expression in the hippocampus - factors that normally restrain inflammatory responses. Conclusions: These findings demon-
\end{abstract}

strate that cellular inflammation is not necessarily suppressed by GCs in the injured hippocampus; instead, GCs may worsen hippocampal neuron death, at least in part by increasing the neurotoxicity of CNS inflammation.

(c) 2014 S. Karger AG, Basel

\section{Introduction}

Glucocorticoid (GC) stress hormones have long been appreciated for their potent anti-inflammatory properties, but closer investigation has revealed that, depending on the context and duration of exposure, GCs can increase some of the same inflammatory responses they normally inhibit [1-5]. In particular, acute GC exposure (from a single stressor) prior to an immune challenge increases leukocyte recruitment to sites of peripheral inflammation in rodents $[6-8]$ and in humans $[9,10]$. This priming effect [11] of GCs switches to the classical immunosuppressive effect if GCs are given after injury [6, 12] or chronically (for weeks) [13]. Timing, concentration, and duration of GC exposure are therefore all critical in determining whether these hormones will be immunoactivating or immunosuppressive $[3,4,14]$, but the

\section{KARGER}

E-Mail karger@karger.com

www.karger.com/nen
(C) 2014 S. Karger AG, Basel

0028-3835/14/1003-0129\$39.50/0
Shawn F. Sorrells

Department of Neurosurgery

Stanford University

Stanford, CA 94305-5020 (USA)

E-Mail sfs11@stanford.edu 
specific situations leading to each outcome are not well documented.

In the CNS, these immune-augmenting GC effects may be detrimental to neuron survival. Subacute or chronic GC exposure that would normally suppress inflammatory responses in the periphery instead lead to increased CNS inflammation in response to bacterial lipopolysaccharide (LPS) $[15,16]$ and excitotoxins $[2,17]$, particularly in glucocorticoid receptor (GR)-rich regions like the frontal cortex and hippocampus. Hallmarks of this increased immune response include increased activity of the proinflammatory transcription factor NF- $\kappa \mathrm{B}$ and a failure of GCs to induce some of their normal anti-inflammatory targets like MKP-1, IL-1ra, and IкBa in the forebrain [1]. In subcortical regions like the hypothalamus or in the periphery, these same GC treatments still lead to a classical decrease in NF- $\kappa \mathrm{B}$ activation [16]. Prolonged GC exposure can lead to compensatory GR downregulation, but immune cell-specific GR knockouts and a GR antagonist or GC synthesis inhibitor can block GC-enhanced inflammation, arguing against this explanation $[2,16,17]$.

Exposure to GCs also impairs neuron survival during many types of acute CNS injury including hypoxia-ischemia, excitotoxicity, and hypoglycemia [18]. GCs impair several processes that are necessary for neurons to survive an injury: the reuptake of excitotoxic glutamate from the synapse [19], sequestration and extrusion of free cytosolic calcium [20], and oxygen radical quenching [21]. Classical anti-inflammatory GC effects would also include suppressing NF- $\kappa \mathrm{B}$ activity [22] and reducing the abundance of proinflammatory mediators like IL-1 $\beta$ [23] and prostaglandins [24]. This might be expected to be neuroprotective because inhibiting IL-1 $\beta$ [25], $\mathrm{PGE}_{2}[26,27]$, or $\mathrm{NF}-\kappa \mathrm{B}$ activation [28] protects against excitotoxic neuron death. As described, however, in some situations GCs can increase the production of these inflammatory mediators, so it could also be the case that GC effects on inflammation further contribute to neuron death.

We therefore sought to investigate whether the effects of GCs on the inflammatory response also contribute to neuron death during excitotoxic injury. To see whether we could manipulate GC-augmented inflammation after injury, we attempted to inhibit it using two anti-inflammatory drugs with different mechanisms of action: indomethacin and minocycline. Indomethacin is a nonselective inhibitor of the COX enzymes that are the rate-limiting step in the production of prostaglandins. Two months of treatment with indomethacin only slightly reduces microglial activation after irradiation [29], so we did not expect this drug to necessarily have a large effect unless COX enzymes were specifically required. In contrast, minocycline is reported to be a potent inhibitor of microglial activation [30].

We present evidence that blocking certain aspects of the inflammatory response can protect neurons from GC endangerment. We also investigate the possibility that GCs condition the CNS in unique ways, priming it to have a greater and potentially more damaging immune response to subsequent injury. The dogma that GCs are only immunosuppressive is being refined as the actions of these hormones are able to be explored with greater contextual resolution.

\section{Materials and Methods}

\section{Experimental Design}

Male Sprague-Dawley rats between 250 and 300 g (Charles River, Gilroy, Calif., USA) were housed in groups of 3 in a 12-hour light-dark cycle and fed ad libitum. Animals were given a minimum of 1 week to acclimate to our vivarium after purchase.

To measure the effects of different GC exposures, rats were anesthetized with $2 \%$ halothane and bilaterally adrenalectomized (ADX) or sutured without ADX (sham). ADX rats were given $100 \mathrm{mg}$ s.c. pellets with either a $15,30,60$, or $100 \%$ corticosterone/cholesterol mixture. These treatments yield blood GC levels within physiological limits $[17,31]$. Rats given $15 \%$ pellets (low GCs) have blood GC levels around 2-4 $\mu \mathrm{g} / \mathrm{dl}$, corresponding with basal hormone levels. Rats given $100 \%$ pellets were also given daily $10 \mathrm{mg} / \mathrm{kg}$ s.c. injections of corticosterone in peanut oil to maintain high blood GC levels between 30 and $40 \mu \mathrm{g} / \mathrm{dl}$. Intermediate 30 and $60 \%$ pellets produce blood hormone levels that correspond with moderate stress, or 15$20 \mu \mathrm{g} / \mathrm{dl}$ and $20-30 \mu \mathrm{g} / \mathrm{dl}[1]$. These animals were compared with each other to isolate the effects of GC concentration, but they were also compared with sham animals that received sham surgery to account for the effects of ADX. For comparison, the injury-induced increase above baseline in peripheral corticosterone levels in sham animals after kainic acid (KA) is $32 \pm 2 \mu \mathrm{g} / \mathrm{dl} 30 \mathrm{~min}$ after injury, $27 \pm 4 \mu \mathrm{g} / \mathrm{dl} 1 \mathrm{~h}$ after injury, $14 \pm 8 \mu \mathrm{g} / \mathrm{dl} 3 \mathrm{~h}$ after injury, $12 \pm 4 \mu \mathrm{g} /$ $\mathrm{dl} 6 \mathrm{~h}$ after injury, and $3 \pm 2 \mu \mathrm{g} / \mathrm{dl} 12 \mathrm{~h}$ after injury $[19,31]$.

To study the effects of GCs on neuron death, we used the KA model of excitotoxicity, where rats are given stereotactic injections of KA into the hilus of the dentate gyrus in the hippocampus. This model leads to reproducible amounts of damage and we previously found that subacute exposure to high GCs (approx. $30 \mu \mathrm{g} / \mathrm{dl}$ ) can increase the inflammatory response to this injury in vivo, with similar effects in primary culture $[17,32]$. After 3 days of exposure to GC treatments, rats were anesthetized with a ketamine/xylazine/acepromazine cocktail $(77 / 7.7 / 1.54 \mathrm{mg} / \mathrm{kg}$, i.p.) and were counterbalanced to first receive either $1 \mu \mathrm{l}$ of phosphate-buffered saline (PBS) in the left hemisphere or $1 \mu \mathrm{l}$ of $0.06 \mu \mathrm{g}$ of KA dissolved in PBS in the right hemisphere at an infusion rate of $0.2 \mu \mathrm{l} /$ min. The coordinates were: $\pm 3.2 \mathrm{~mm}$ lateral, $4.5 \mathrm{~mm}$ posterior to the bregma, $3.3 \mathrm{~mm}$ ventral to the dura. Buprenorphine $(0.03 \mathrm{mg} /$ $\mathrm{kg}$, s.c.) was given as a postoperative analgesic. In animals receiving additional drug treatments, indomethacin $(10 \mathrm{mg} / \mathrm{kg}$ i.p. $)$ was injected $24 \mathrm{~h}$ prior to KA and every $12 \mathrm{~h}$ thereafter until the rats were 
killed. Minocycline ( $22.5 \mathrm{mg} / \mathrm{kg}$ s.c.) was given $30 \mathrm{~min}$ prior to $\mathrm{KA}$, and daily for the next $72 \mathrm{~h}$. All drugs were purchased from Sigma (St. Louis, Mo., USA). Unless otherwise indicated, the rats were sacrificed 2-4 h after lights on. All rats were treated in accordance with the Stanford University Administrative Panel on Laboratory Care regulations and the NIH Guide for the Care and Use of Laboratory Animals.

\section{Lesion Quantification}

Rats were transcardially perfused $72 \mathrm{~h}$ after KA with $0.9 \%$ saline followed by $4 \%$ PFA. Brains were postfixed in 4\% PFA and 30\% sucrose for $24 \mathrm{~h}$ prior to cutting $20-\mu \mathrm{m}$ frozen cryostat sections. Sections were stained with cresyl violet, and $40 \times$ images of the CA3 region were acquired using an Olympus IX70 microscope and Hamamatsu digital camera. CA3 damage was calculated by dividing the remaining CA3 cell area in the KA-injected hemisphere by that of the contralateral PBS-injected hemisphere as previously described [17].

\section{Immunohistochemistry}

Sections were air-dried for $20 \mathrm{~min}$, blocked in 5\% normal goat serum in PBS $+0.3 \%$ Triton-X 100 (PBST) for $1 \mathrm{~h}$ at room temperature, and incubated overnight at $4^{\circ} \mathrm{C}$ with mouse anti-rat $\mathrm{CD} 11 \mathrm{~b} / \mathrm{c}$ at 1:100 dilution in PBST (OX-42 clone, Serotec). Sections were washed in PBST $3 \times$ for $10 \mathrm{~min}$ and incubated for $1 \mathrm{~h}$ at room temperature with goat anti-mouse Alexa 488 (1:200 in PBST, Invitrogen). Sections were washed again in PBST $3 \times$ for $10 \mathrm{~min}$ and stored in polyvinyl alcohol with DABCO (Sigma). Metamorph (Universal Imaging) was used to quantify the amount of CD11b/c signal according to guidelines for quantifying fluorescent signals [30]. Briefly, 5 evenly spaced sections from the hippocampus of each rat were stained and uniformly background corrected. Signal was only included if it was 10 pixel intensity units above background and between 3 and $17 \mu \mathrm{m}$ in diameter. These parameters were empirically determined based on the average size of microglia cell bodies [33] to eliminate autofluorescent debris and artifacts. Total signal estimates are therefore not an approximation of microglia number, but are an approximation of the area they encompass [34].

\section{Quantitative PCR}

Total RNA was extracted using the RNEasy Plus Mini Kit (Qiagen), and was reverse-transcribed using the iScript cDNA synthesis kit (BioRad). Intron-spanning primers used were (sense, antisense): CX3CL1 (NM_134455): 5'-GCATGACGAAATGCAA CATC-3' 5' $^{\prime}$-TTGGACCCATTTCTCCTTTG-3'; CD22 (NM_00 1107503): $5^{\prime}$-CTGTGGCCGTGGAGATAGAT-3'， 5'-AAGAG GGTTTGGGGATGTTC-3'; CD200 (NM_031518): 5'-TGTTT GGATCTGGGAAGGTC-3', 5'-GGAGGATGCTGGTGACAG AT-3'; TGF- $\beta 1$ (NM_021578): 5'-GCGGACTACTACGCCA AAGA-3', 5' ${ }^{\prime}$-CGTGTTGCTCCACAGTTGAC-3', and IL-1ra (NM_001039701): 5'-GAAAAGACCCTGCAAGATGC-3' ${ }^{\prime}, 5^{\prime}$ GATGCCCAAGAACACATTCC-3'. Quantitative PCR was performed on a BioRad PCR machine using SyBRGreen and PCR Miner software [35].

\section{Electrophoretic Mobility Shift Assay}

Following GC treatment, the CA3 was microdissected and nuclear extracts were prepared [16]. Electrophoretic mobility shift assay to NF- $\kappa B$ was performed using the gel shift assay kit from Promega and the consensus oligonucleotide probe $\left(5^{\prime}\right.$-AGTT

GCs Worsen CNS Inflammation and Excitotoxicity
GAGGGGACTTTCCCAGGC-3') [36]. Autoradiographs of nondenaturing acrylamide electrophoresis were quantified with the ChemImager detection system (Alpha-Innotech, San Leandro, Calif., USA), and several exposure times to ensure linearity of band intensities.

\section{ELISAs}

Protein was prepared from dissected CA3 tissue in NP-40 lysis buffer (150 mM sodium chloride, 1.0\% Nonidet-P40, $50 \mathrm{~mm}$ Tris, $\mathrm{pH}$ 8.0) and quantified by the BCA method (Pierce). ELISAs for MCP-1, CINC-1, and IL-1b (R\&D) were run as per manufacturer instructions and quantified on a Molecular Devices plate reader. No values fell outside of the manufacturer-reported limits for these ELISAs: MCP-1: $10 \mathrm{pg} / \mathrm{ml}$, CINC-1: $1.3 \mathrm{pg} / \mathrm{ml}$, and IL- $1 \beta: 5 \mathrm{pg} / \mathrm{ml}$.

\section{Statistics}

Data are presented as means \pm SEM. Statistical tests and sample sizes used to establish significance are indicated in figure legends. All statistical tests were performed using GraphPad Prism.

\section{Results}

\section{GC-Augmented Immune Cell Activation in the} Injured Hippocampus Is Blocked by Minocycline but Not Indomethacin

We selected two different anti-inflammatory drugs that block different components of the inflammatory response to injury. Rats were given injections of either indomethacin or minocycline concurrent with KA-induced excitotoxic injury (fig. 1a). Three days after injury, the animals were killed and brain sections were stained for activated infiltrating and resident immunecells $(\mathrm{CD} 11 \mathrm{~b} / \mathrm{c})$ in the injury site (fig. $1 \mathrm{~b}, \mathrm{c}$ ). The same sections were also co-stained with GFAP to indicate the area of gliosis resulting from the injury (fig. 2).

Low GC animals had approximately $50 \%$ of the $\mathrm{CD} 11 \mathrm{~b} / \mathrm{c}+$ area in sham animals. This decrease, although not significant, was significantly increased in animals given high GCs to an amount of $\mathrm{CD} 1 \mathrm{~b} / \mathrm{c}+$ area similar to that observed in sham animals. Concurrent treatment with the nonselective COX inhibitor indomethacin had no significant effects on CD11b/c signal in any of the groups. In contrast, minocycline, an inhibitor of NF- $\kappa \mathrm{B}$ and microglia activation [30, 37, 38], significantly reduced CD11b/c signal in both sham and high GC animals. The amount of GFAP signal was similar between all groups, but was reduced by approximately $50 \%$ in animals receiving minocycline (fig. $2 \mathrm{~b}$ ). These results demonstrate that elevated GC concentrations are sufficient to increase inflammatory cell activation and show that these increases are sensitive to minocycline but not indomethacin treatment. 
Fig. 1. CD11b/c+ area is increased by GCs and reversed by minocycline following $\mathrm{KA}$ injury. a Experimental design: male rats were given 1 week to acclimate after arriving at our animal facility. GC manipulations were performed 3 days prior to KA, and brains were analyzed 3 days later. Indomethacin was injected every $12 \mathrm{~h}$ starting $24 \mathrm{~h}$ before KA. Minocycline was injected every $24 \mathrm{~h}$ starting $30 \mathrm{~min}$ before KA. b Representative images of CD11b/c staining in the hippocampal CA3 region $72 \mathrm{~h}$ after injury. Scale bars $=500 \mu \mathrm{m}, 100 \mu \mathrm{m}$ inset. c The total amount of CD $11 \mathrm{~b} / \mathrm{c}+$ area was significantly lower in low GC rats compared with high GC rats. Minocycline significantly reduced CD $11 \mathrm{~b} / \mathrm{c}$ signal in sham rats and high GC rats, but not low GC rats. Significance levels ${ }^{*} \mathrm{p}<0.05$ and ${ }^{* *} \mathrm{p}<0.01$ established by 2-way ANOVA followed by a Holm-Sidak post hoc test. Significant 2-way ANOVA main effect of GC treatment $(\mathrm{p}<0.05)$ and drug treatment $(\mathrm{p}<$ $0.0001)$. Sham, vehicle $n=7$; low GC, vehicle $\mathrm{n}=12$; high GC, vehicle $\mathrm{n}=6$; sham, indomethacin $n=6$; low GC, indomethacin $\mathrm{n}=5$; high GC, indomethacin $\mathrm{n}=9$; sham, minocycline $\mathrm{n}=7$; low GC, minocycline $\mathrm{n}=7$; high $\mathrm{GC}$, minocycline $\mathrm{n}=6$.

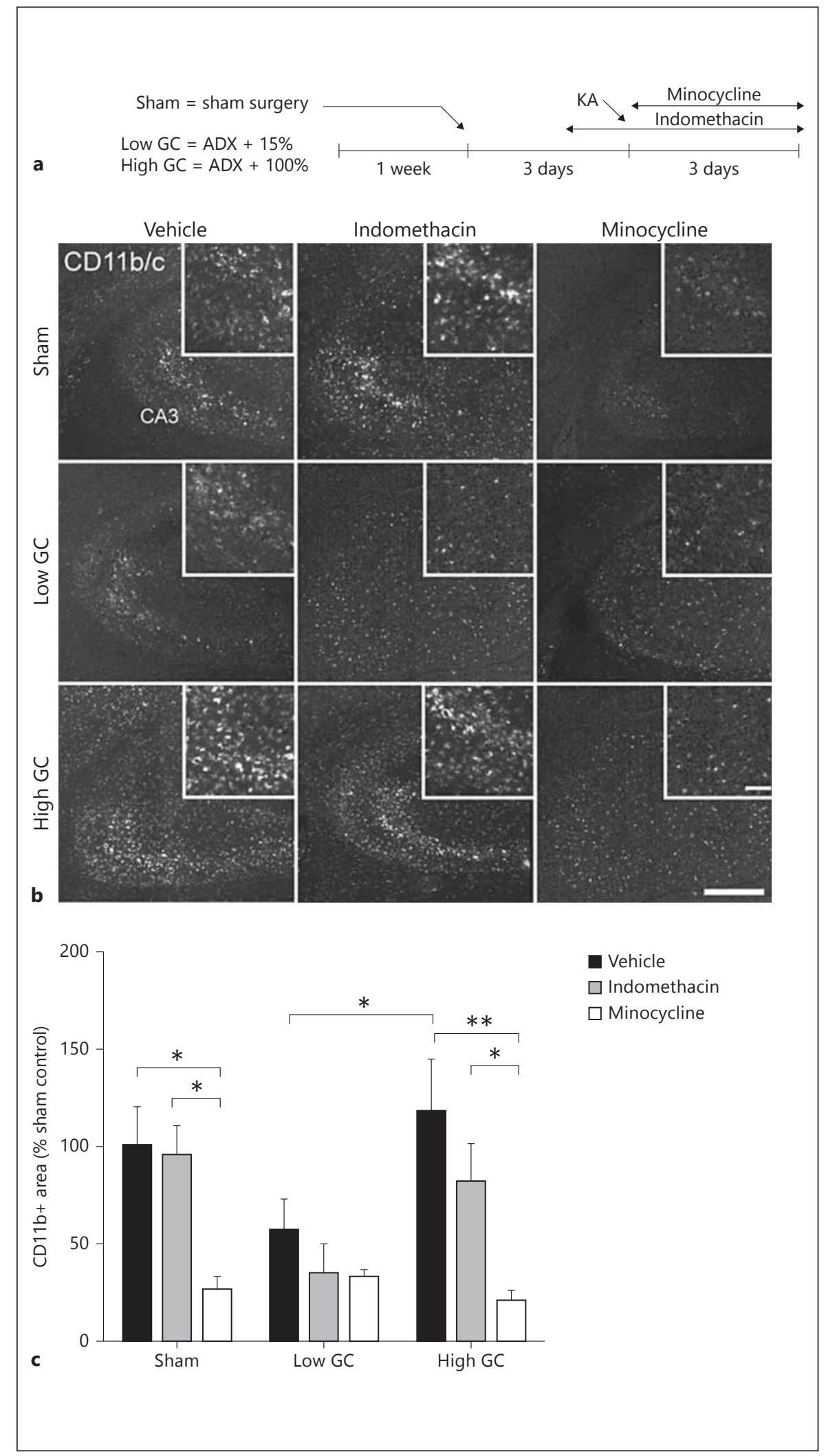


Fig. 2. GFAP+ area is reduced by minocycline following KA injury. a Representative images of GFAP staining in the hippocampal CA3 region $72 \mathrm{~h}$ after injury. Scale bars $=500 \mu \mathrm{m}, 100 \mu \mathrm{m}$ inset. $\mathbf{b}$ The total amount of GFAP+ area was reduced by minocycline treatment, but not by indomethacin treatment or GC manipulations. Significance levels $* \mathrm{p}<0.05$ and ${ }^{* *} \mathrm{p}<0.01$ established by 2-way ANOVA followed by a Holm-Sidak post hoc test. Significant 2-way ANOVA main effect of drug treatment $(\mathrm{p}<0.0001)$. Sham, vehicle $\mathrm{n}=7$; low $\mathrm{GC}$, vehicle $\mathrm{n}=12$; high $\mathrm{GC}$, vehicle $\mathrm{n}=6$; sham, indomethacin $\mathrm{n}=6$; low GC, indomethacin $\mathrm{n}=5$; high GC, indomethacin $\mathrm{n}=9$; sham, minocycline $\mathrm{n}=7$; low $\mathrm{GC}$, minocycline $\mathrm{n}=7$; high $\mathrm{GC}$, minocycline $\mathrm{n}=6$.

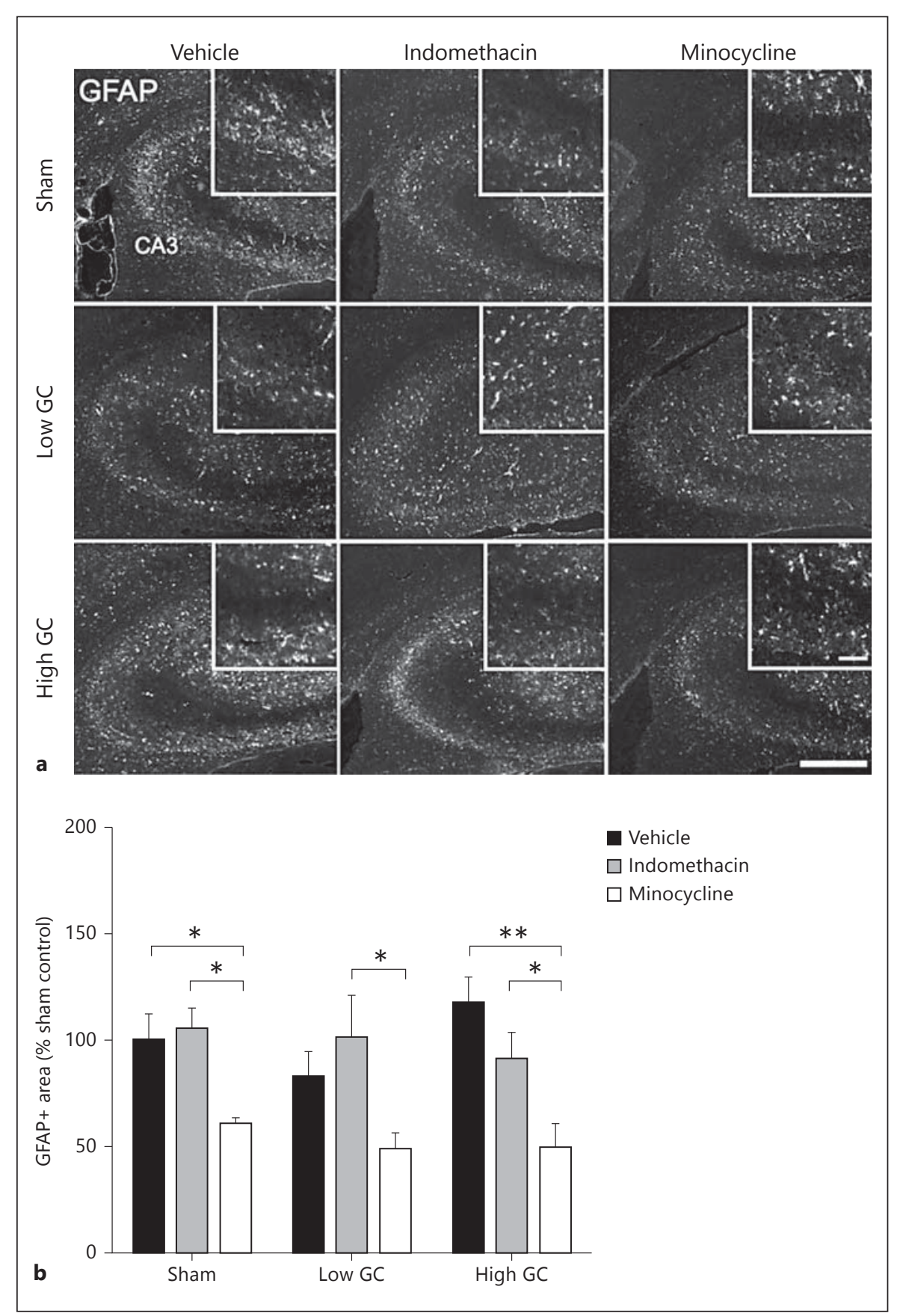

\section{GC-Augmented Cellular Inflammation Impairs}

Neuron Survival

We next measured whether these anti-inflammatory drug treatments could protect neurons from GC endangerment. To quantify neuron death, the damaged area in the CA3 hippocampal field was measured in subsequent tissue sections (fig. 3a, arrows). The dose of KA delivered to the hilus of the dentate gyrus resulted in approximate- ly $50 \%$ CA3 cell loss. In agreement with previous examples of GC endangerment [17, 31, 39], high GCs significantly worsened CA3 neuron death (fig. 3b). Indomethacin was unable to protect CA3 neurons from this GC endangerment, but minocycline restored CA3 damage to levels observed in sham animals. This result is consistent with the differing ability of these drugs to block the cellular response to injury, and supports the hypothesis that 


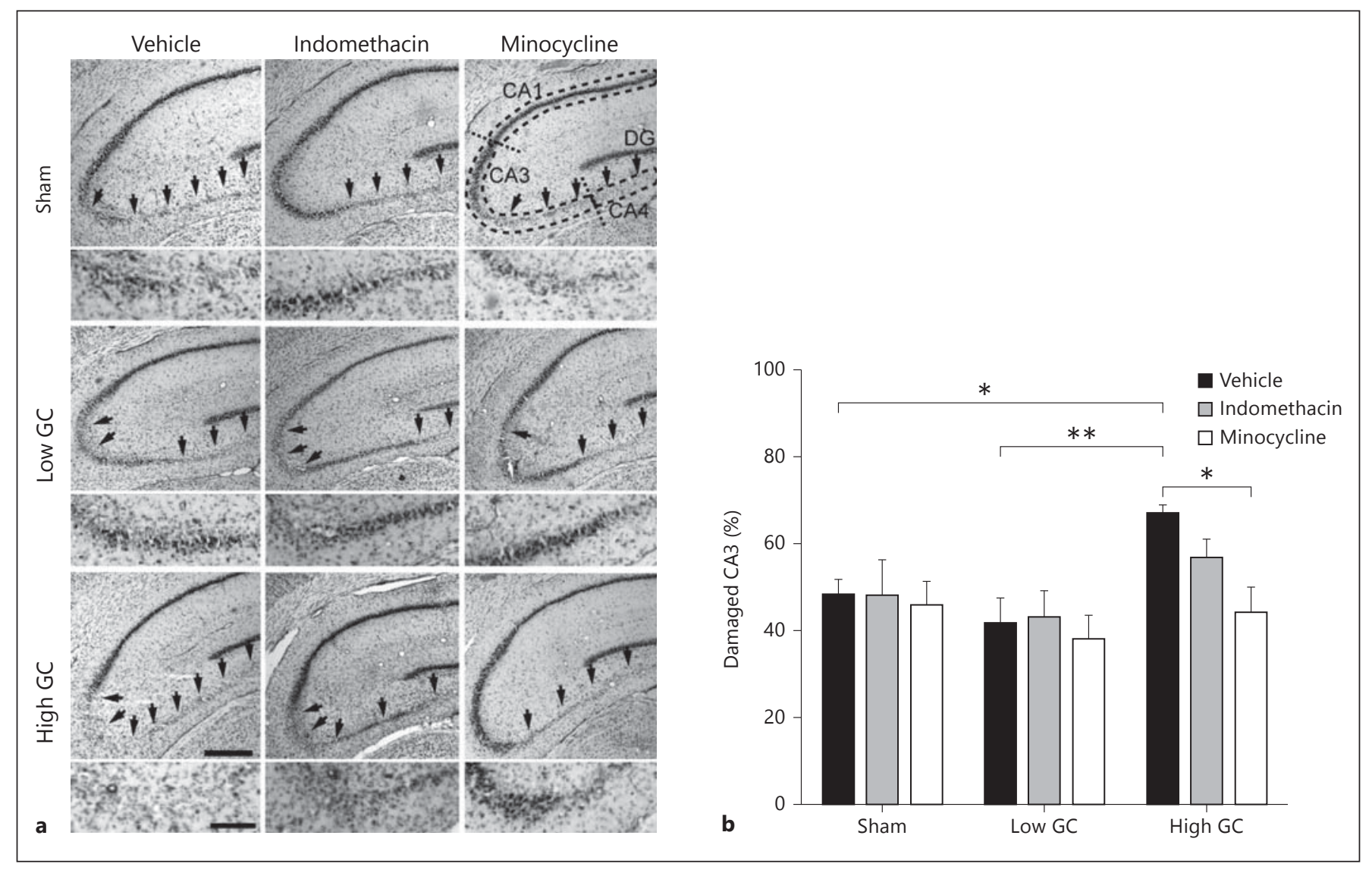

Fig. 3. High GCs increase excitotoxic injury which is reversed by minocycline. a Representative images of Nissl-stained sections following each treatment combination $72 \mathrm{~h}$ after KA. Damage was quantified in the CA3 region (magnified below each image). Hippocampal subregions are indicated with dashed lines. Arrows point to areas of neuron loss. Scale bars $=500 \mu \mathrm{m}(200 \mu \mathrm{m}$, magnification). b Quantification of the CA3-lesioned area $72 \mathrm{~h}$ after injury. High GCs significantly increased CA3 damage relative to

augmented inflammatory responses contribute to GC endangerment of neurons. Importantly, we did not observe a neuroprotective effect of reducing inflammation in all of the groups (e.g. low GC animals). We next sought to improve our understanding of how these GC manipulations alter postinjury inflammation.

\section{GCs Dose-Dependently Influence Chemokine Release after Injury}

One possible explanation for GC-enhanced immune activation is that GCs increase the signals that activate or recruit immune cells. The injured CA3 region was microdissected at several time points after injury to measure the levels of two principal chemokines involved in recruiting monocytes (CCL2) and granulocytes low GCs and sham rats. This was reversed by minocycline, but not indomethacin treatment. Significance levels $* \mathrm{p}<0.05$ and ${ }^{* *} \mathrm{p}<$ 0.01 established by 2 -way ANOVA followed by a Holm-Sidak post hoc test. Significant 2-way ANOVA main effect of GC treatment $(\mathrm{p}<0.01)$. Sham, vehicle $\mathrm{n}=7$; low $\mathrm{GC}$, vehicle $\mathrm{n}=12$; high $\mathrm{GC}$, vehicle $n=6$; sham, indomethacin $n=6$; low $\mathrm{GC}$, indomethacin $\mathrm{n}=5$; high GC, indomethacin $\mathrm{n}=9$; sham, minocycline $\mathrm{n}=7$; low GC, minocycline $\mathrm{n}=7$; high GC, minocycline $\mathrm{n}=6$.

(CINC-1) (fig. 4). A careful time-course revealed that both low and high GC animals had earlier CCL2 and higher CINC-1 levels in the injury site (fig. 4a, c). For CINC-1, the chemokine release was also sharper, increasing from and decreasing to levels well below those measured in sham animals. Both chemokine levels had nearly returned to baseline by $48 \mathrm{~h}$ after injury. Because both low and high GC groups responded similarly, we wondered if these were effects of ADX, so we picked the 8-hour time point to compare intermediate GC concentrations of 30 and $60 \%$ (fig. 4b, d). Chemokine levels at this time after injury revealed a U-shaped dose response to GC hormone levels. A nonlinear fit of a second-order polynomial curve produced $\mathrm{R}^{2}$ values of 0.89 for CCL2 and 0.82 for CINC- 1 . 

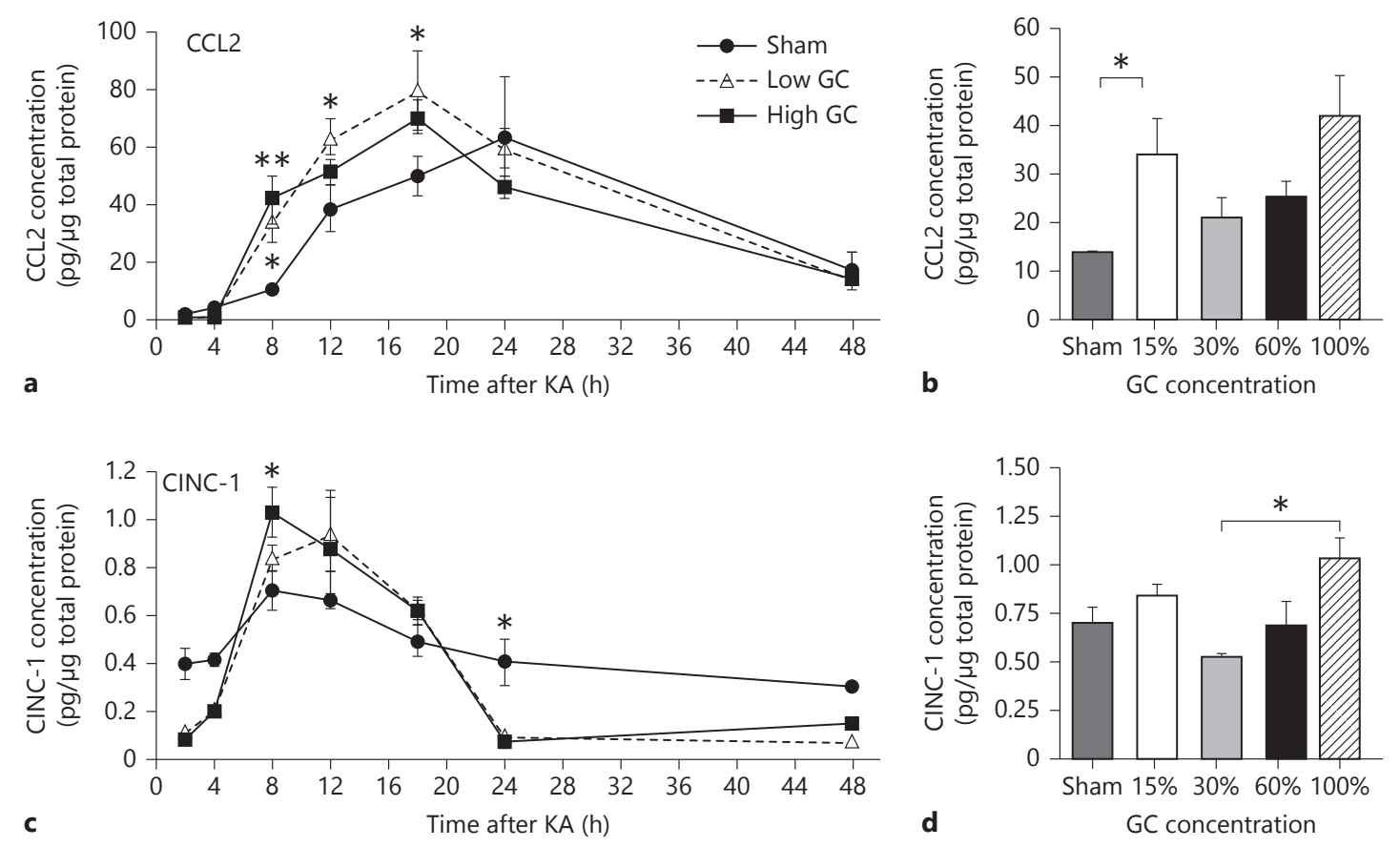

Fig. 4. GCs alter chemokine release after injury. CA3 tissue was collected at $2,4,8,12,18,24$, and $48 \mathrm{~h}$ after KA injury. $\mathrm{n}=3-6$ per group. a CCL2 production peaked $18 \mathrm{~h}$ after KA and was significantly elevated over sham animals in both the low GC and high GC groups at $8 \mathrm{~h}$ and continued to be elevated in low GC animals from 18 to 24 h. Significance levels $* p<0.05$ and ${ }^{* *} p<0.01$ established by 2-way ANOVA followed by a Holm-Sidak post hoc test. Significant 2-way ANOVA main effect of time after injury ( $<<0.0001$ ). b CCL2 production $8 \mathrm{~h}$ after injury was significantly higher in low GC animals compared to sham. Significance established by 1-way

These results suggest that GCs can alter chemokine levels with a classical U-shaped dose response, but also that the large increases in inflammatory cell activation after injury are not due to a corresponding large increase in chemokine signaling.

\section{GCs Suppress Anti-Inflammatory Signals in the Hippocampus prior to Injury}

Based on the hypothesis that GCs might prime the CNS to be hyperresponsive to subsequent injury [6], we measured the effects of exposure to these GC treatments on the expression of several genes that keep the immune response in check within the CNS. Neurons produce multiple signals that suppress microglia activation, including CX3CL1, CD200, and CD22 [40-43]. We also examined TGF- $\beta 1$ because GCs normally induce this gene as a component of their anti-inflammatory actions $[22,44]$. To de-
ANOVA followed by a Holm-Sidak post hoc test. Significant 1-way ANOVA main effect $(\mathrm{p}<0.05)$. c CINC-1 production peaked $8 \mathrm{~h}$ after KA and was increased in high GC animals relative to sham. Significance established by 2-way ANOVA followed by a HolmSidak post hoc test. Significant 2-way ANOVA main effect of time after injury $(\mathrm{p}<0.0001)$ and significant interaction $(\mathrm{p}<0.01)$. d CINC-1 production $8 \mathrm{~h}$ after injury was significantly higher in animals given $100 \%$ GC pellets compared to those given $30 \%$. Significance established by 1-way ANOVA followed by a Holm-Sidak post hoc test. Significant 1-way ANOVA main effect $(\mathrm{p}<0.01)$. termine whether GCs alter the expression of these genes, their levels were measured by qPCR at the time when KA injury was previously given (fig. 5a).

GCs significantly altered the expression of several of these anti-inflammatory signaling molecules in the hippocampus. Low GC rats had 2- to 4 -fold increases in the expression of CX3CL1, its receptor CX3CR1, and CD22 relative to either sham or high GC rats (fig. 5a). This suggested that removing GCs by ADX leads to a compensatory increase in these anti-inflammatory signaling molecules. Furthermore, this increase could be reversed in ADX animals by treatment with high GCs, suggesting that GCs might be the necessary factor removed by ADX. Because GCs suppressed these antiinflammatory signals, we next examined whether this resulted in an increase in proinflammatory mediators prior to injury. 
Fig. 5. GCs suppress anti-inflammatory gene expression. Rats were treated with GCs and the hippocampus was dissected at the time when the injury would normally be given. $n=5-6$ per group. a Anti-inflammatory gene expression in the uninjured hippocampus following each GC treatment, expressed as fold-increase relative to sham. Low GC animals had elevated CX3CL1, CX3CR1, and CD22 expression relative to sham or high GC-treated animals $(* \mathrm{p}<$ 0.05 , $^{* *} \mathrm{p}<0.01,{ }^{* * *} \mathrm{p}<0.001, * * * * \mathrm{p}<$ $0.0001)$. Significance established by 2 -way ANOVA followed by a Holm-Sidak post hoc test. b IL- $1 \beta$ protein levels in the uninjured hippocampus following each GC treatment. High GC animals had higher IL$1 \beta$ levels than low GC animals $(* \mathrm{p}<0.05)$. Significance established by a 2-way unpaired t test. c Quantification of electrophoretic mobility shift assay autoradiographs for the p65 subunit of NF- $\mathrm{KB}$ in the uninjured hippocampus. Basal NF- $\kappa \mathrm{B}$ activation was not affected by GC treatments $\left(^{*} \mathrm{p}<\right.$ $\left.0.05,{ }^{* *} \mathrm{p}<0.01,{ }^{* * * *} \mathrm{p}<0.0001\right)$. Significance established by 2-way ANOVA followed by a Holm-Sidak post hoc test.

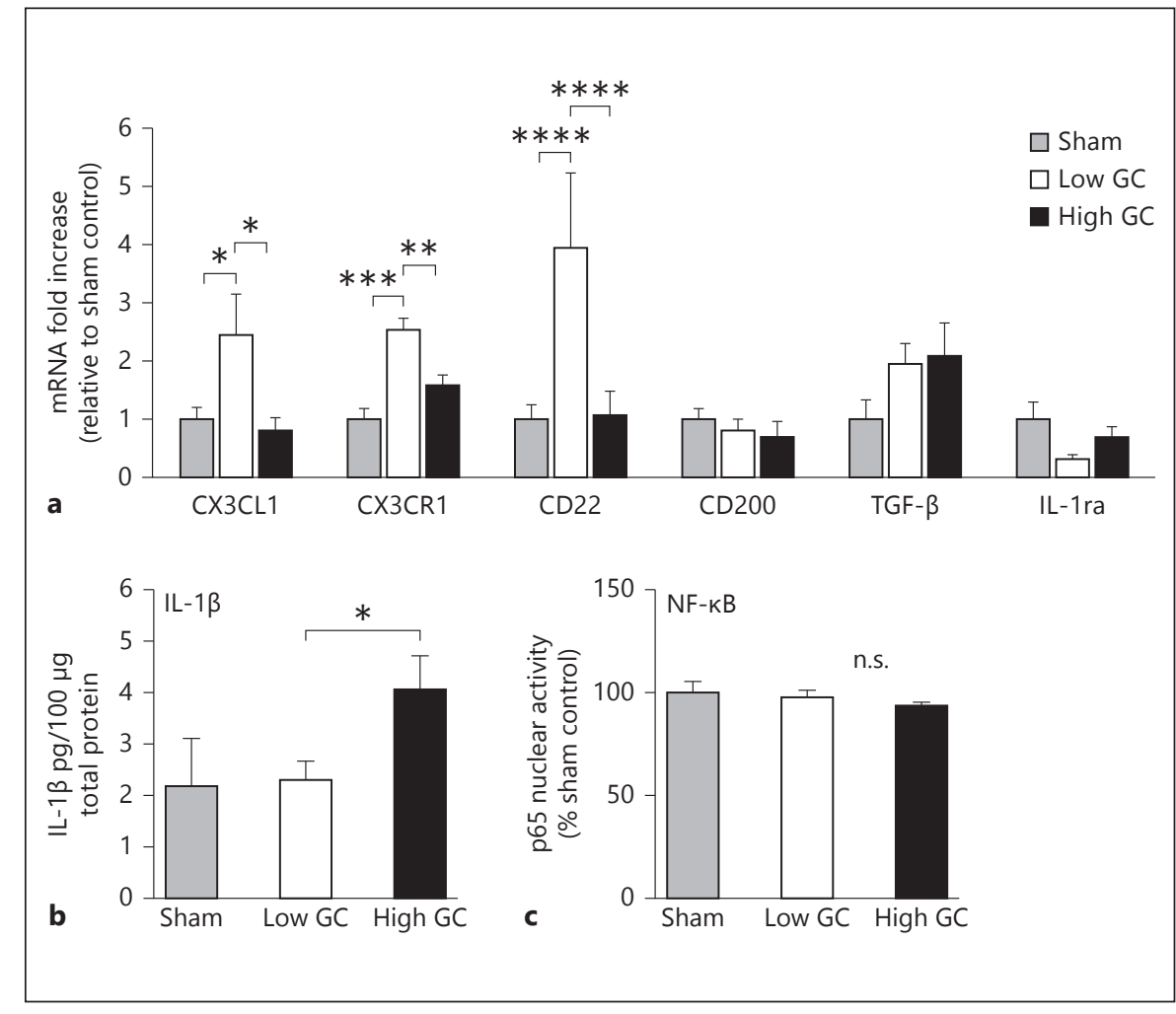

GCs Increase Proinflammatory Signals in the

Hippocampus prior to Injury

Previous reports of GC-augmented inflammation have detected increased production of the proinflammatory cytokine IL- $1 \beta$ in response to an inflammatory challenge $[1,15-17,32]$. If GCs also increase the production of this cytokine prior to the injury, it might lead to augmented and potentially more neurotoxic immune responses, particularly in the context of reduced anti-inflammatory signaling by molecules like CX3CL1 [45].

Although IL- $1 \beta$ levels were quite low in the uninjured hippocampus, high GC animals had significantly more IL- $1 \beta$ than low GC rats (fig. $5 b$ ). Because IL- $1 \beta$ production is stimulated by $\mathrm{NF}-\kappa \mathrm{B}$ transcriptional activity, and NF- $\mathrm{kB}$ is normally a target of the antiinflammatory actions of GCs, we next investigated whether the increased IL-1 $\beta$ might result from increased NF- $\kappa \mathrm{B}$ activity. We measured the DNA-binding activity of the p65 subunit of NF- $\mathrm{kB}$ via electrophoretic mobility shift assay following each GC treatment, but there were no differences in DNA binding activity (fig. $5 c$ ), suggesting that the IL- $1 \beta$ increase was NF- $\kappa B$ independent.

\section{Discussion}

Our findings suggest that GCs can increase cellular inflammatory responses to excitotoxic injury in the hippocampus and we identified several anti-inflammatory signaling molecules that are suppressed by GCs and could contribute to an augmented cellular response. This increased inflammation was detrimental to the ability of neurons to survive excitotoxic injury and represents a new mechanism whereby GCs can endanger neurons.

\section{GCs Increase Immune Cell Activation in the Injured Hippocampus}

Comparison of low and high GC rats revealed that high GC concentrations increased the CD11b/c+ area. This agrees with previous reports that GCs can increase immune cell activation both basally [46] and in this same excitotoxicity model [17]. Previous work using this same excitotoxicity model found that ADX decreased inflammatory cell activation and sham rats given additional GCs had higher inflammatory cell activation [17]. These effects could potentially be due to compensatory decreases in endogenous GC synthesis (comparing sham with sham given additional GCs) or they could be an artifact of ADX 
(comparing sham with $\mathrm{ADX}$ ). The present finding that GC-augmented inflammation still occurs when the GC dose is an isolated variable in ADX rats argues against these alternative possibilities.

The different ability of the two drugs to inhibit GCaugmented cellular activation also narrows the possible mechanisms responsible. The ineffectiveness of indomethacin argues that prostaglandin synthesis is not necessary for GCs to increase inflammation. While it was expected that minocycline would generally reduce inflammatory cell activation, it does this at least in part by inhibiting NF- $\kappa \mathrm{B}$ activation [30], and this is the same target of normal GC anti-inflammatory effects. If GCs were already inhibiting NF- $\kappa \mathrm{B}$, minocycline would be expected to be less effective. Instead, we found the opposite: minocycline suppressed immune cell activation in sham and high GC rats, but could not further reduce cellular activation in low GC rats. Thus we conclude that GCs increase immune cell activation in the injured hippocampus and minocycline can reverse these effects, whereas indomethacin cannot.

\section{GCs Do Not Augment Inflammation via Increased Chemokine Release}

Given that GCs doubled immune cell activation, a likely possibility is that they do so by accelerating and/or increasing the magnitude of chemokine release in the injury site. The predominant chemokine source is inflammatory cells themselves, so increased cellular activation in the presence of GCs might correspond with higher chemokine levels. Instead, we found that postinjury CCL2 and CINC-1 levels were only slightly changed by GC treatments (fig. 4), suggesting either that GCs are suppressing chemokine production or that greater cellular activation does not equate with more chemokine production. From this we concluded that GCs must affect other mechanisms to increase immune activation.

\section{GCs Suppress Anti-inflammatory Signals in the} Hippocampus prior to Injury

We asked whether GCs suppress the expression of anti-inflammatory genes and found that they did affect several genes in this way (fig. 5a). The anti-inflammatory genes that GCs suppress are at least partially redundant in their ability to restrain the immune response to injury. CX3CR1 is a receptor present on immune cells, and its ligand CX3CL1 is a soluble factor expressed in neurons throughout the CNS [47]. CX3CR1 knockout mice have a more neurotoxic microglial response to a number of different CNS injuries [45]. CD22 is less extensively studied, but is a soluble ligand for the panleukocyte marker CD45 and it inhibits microglial release of cytokines in response to LPS [42]. That GCs affect these molecules in the CNS is not unprecedented as they have been seen to reduce CX3CL1 expression in respiratory epithelial cells [48]. In peripheral injury, GCs suppress CX3CL1 expression by displacing NF- $\kappa \mathrm{B}$ from the CX3CL1 promoter [48]; however, in the absence of a stimulus, NF- $\kappa B$ is not present on this promoter, suggesting that GCs must be able to suppress CX3CL1 via an alternative method and that GC suppression of CX3CL1 expression might be even greater after injury. Thus, exposure to GCs reduces the expression of several critical inhibitory inflammatory signals in the hippocampus, in agreement with our findings in immune-cell GR knockout mice [2].

GC-mediated suppression of these anti-inflammatory genes corresponds with the amount of cellular activation post-injury (fig. 1b). Low GC rats have one half the immune activation of high GC rats and have correspondingly elevated anti-inflammatory gene expression. Because high GCs did not suppress anti-inflammatory genes beyond the level of sham rats, it could be that GC signaling is required in a permissive fashion to facilitate an appropriately robust inflammatory response. It is possible that if sham rats were given high GC treatment, these genes might be even further suppressed since sham rats given additional GCs have greater hippocampal immune cell responses to excitotoxicity than sham rats alone [17].

We also measured GC effects on the proinflammatory cytokine IL-1 $\beta$ in the absence of injury. This cytokine is increased by GCs in the uninjured hippocampus [17] and after generalized inflammation from peripheral or centrally injected LPS $[1,15,16]$, or excitotoxicity $[17,34]$. In the present work, high GC animals had twice the IL- $1 \beta$ protein levels of low GC animals in the uninjured hippocampus (fig. 5b). High GCs should suppress IL- $1 \beta$ relative to low if GCs were acting in their normal anti-inflammatory manner. This suggested that in addition to suppressing anti-inflammatory signals, GCs might also have a deficit in their normal ability to inhibit proinflammatory signals. Supporting this, GCs failed to induce IL-1ra, the anti-inflammatory antagonist of IL-1 $\beta$ (fig. $5 \mathrm{a}$ ). To further test this hypothesis we measured the ability of GCs to inhibit NF- $\mathrm{KB}$, a normal target of their anti-inflammatory actions. GC treatment did not affect basal $\mathrm{NF}-\mathrm{\kappa B}$ activation (fig. $5 \mathrm{c}$ ), indicating that the cytokine changes prior to injury were not associated with changes in NF- $\kappa$ B DNA-binding activity.

These results narrow down the possible causes of the GC-augmented cellular response. It is not due to GCs in- 
creasing the production of chemokines in the injury site. Instead, GCs suppress the expression of several important anti-inflammatory genes in a manner that could lead to increased immune activation during a subsequent injury. Furthermore, GCs have an impaired ability to inhibit NF- $\kappa B$ in the hippocampus and fail to induce some of their normal anti-inflammatory targets. Although we did not determine whether GR or mineralocorticoid receptors are important for these effects, these changes were observed in the high GC group relative to the low GC group, suggesting that the increased occupancy of the GR could be responsible. Additionally, previous work has demonstrated reversal of GC-augmented inflammation by mifepristone administration (blocking GR) [16]. There is some precedent for GCs having concentration-dependent effects via mechanisms other than the often-opposing effects of GRs and mineralocorticoid receptor activation. While GCs can potently suppress proinflammatory cytokines after injury, they also simultaneously increase the expression of the corresponding cytokine receptor [49]. This results in minimal cytokine signaling at extreme GC concentrations and peak signaling at an intermediate GC concentration. This could serve to optimize the course of inflammation after injury; however, it remains an open question whether prior exposure to high GCs affects the receptors for these inflammatory mediators in the same manner as endogenous GC release after injury.

\section{GC Effects on Inflammation Contribute to GC \\ Endangerment of Neurons}

These results suggest that the effects of GCs on inflammation might be detrimental to neurons. The reduced ability of GCs to inhibit proinflammatory signals like IL$1 \beta$ in the hippocampus could imperil neurons because increased neurotoxicity of microglia lacking CX3CR1 is mediated via IL- $1 \beta$ [45]. Furthermore, NF- $\kappa$ B activation in immune cells during excitotoxicity increases neuron death [28] and GCs did not reduce NF- $\mathrm{KB}$ activity.

Treatment with minocycline blocked GC endangerment, which supports the argument that GC-augmented inflammation is necessary for GCs to endanger neurons. Indomethacin did not reduce immune activation and was also unable to protect neurons. However, GC-augmented inflammation alone is not sufficient to endanger neurons because minocycline also suppressed cellular responses in sham rats, but was not neuroprotective, and low GC animals had reduced cellular activation but no neuroprotection. The fact that decreasing the cellular inflammation in the context of high GCs was protective argues that
GCs either make neurons more vulnerable to the potential toxicity of an augmented inflammatory response or increase the toxicity of that response itself. Minocycline can also have anti-apoptotic effects, but GCs do not endanger neurons by increasing apoptosis [50], making this an unlikely explanation for the effectiveness of minocycline. Thus, GC effects on inflammation are likely to contribute, at least in part, to GC endangerment.

During the energy crisis caused by excessive excitatory neurotransmitter release, GCs impair multiple processes including glucose transport, glutamate reuptake, calcium sequestration, and the scavenging of reactive oxygen species [18]. None of these effects can individually account for GC endangerment, supporting the notion that GC endangerment emerges from the varied effects of these hormones. The findings we report here add an additional source of endangerment as the anti-inflammatory properties of GCs might have normally been expected to be protective.

\section{Conclusion}

GC-augmented inflammation in the hippocampus is therefore likely to worsen neuron death and is not simply a consequence of GCs making more neurons die. This fits with the finding that the amount of GC-augmented immune cell activation prior to (but not after) the emergence of damage predicts the extent of subsequent GC endangerment [17]. Furthermore, GCs can also increase inflammation in the hippocampus in response to a peripheral inflammatory challenge that causes no neuron death (i.e. LPS) $[1,16]$. GCs can also augment inflammation in humans in a pattern consistent with rodent studies. GC treatments prior to LPS challenge in humans potentiate plasma IL-6 and TNF- $\alpha$ levels $[9,51]$. Moreover, even pharmacological GCs like dexamethasone have been reported to induce the expression of factors associated with innate immune responses (amid decreasing components of adaptive immunity) in human blood mononuclear cells [10].

These findings demonstrate a functional role for GCaugmented inflammation in CNS injury. Future studies are required to verify the physiological relevance of the injury model in this study and to further identify the molecular changes associated with exposure to high GCs. One critical caveat is the artificial nature of our glucocorticoid manipulations. Despite this, we conclude that exposure to high levels of GCs for as little as a few days prior to hippocampal injury is sufficient to increase 
cellular and molecular inflammatory responses with detrimental consequences for neuron survival. These results support the idea that prior GC exposure modifies the immune environment in the hippocampus making it more toxic to injured neurons, and could reflect a unique response of the hippocampus to GCs $[1,16]$.

\section{Acknowledgements}

The authors thank Angela Lee, Javier Caso, Marta Perez, Trevor Sorrells, Norman Ruby, Jessie Ansari, and Kevin Kinney for help with experiments and discussions about the manuscript. This work was supported by NIH F31 NS063491 (S.F.S.), and NIH R01 NS059918 (R.M.S.). The authors declare no conflict of interest.

\section{References}

1 Munhoz CD, Sorrells SF, Caso JR, Scavone C, Sapolsky RM: Glucocorticoids exacerbate lipopolysaccharide-induced signaling in the frontal cortex and hippocampus in a dose-dependent manner. J Neurosci 2010;30:1369013698.

2 Sorrells SF, Caso JR, Munhoz CD, Hu CK, Tran KV, Miguel ZD, Chien BY, Sapolsky RM: Glucocorticoid signaling in myeloid cells worsens acute CNS injury and inflammation. J Neurosci 2013;33:7877-7889.

- 3 Sorrells SF, Caso JR, Munhoz CD, Sapolsky RM: The stressed CNS: when glucocorticoids aggravate inflammation. Neuron 2009;64:3339.

4 Dhabhar FS: Enhancing versus suppressive effects of stress on immune function: Implications for immunoprotection and immunopathology. Neuroimmunomodulation 2009;16: 300-317.

$\checkmark 5$ Yeager MP, Guyre PM, Munck AU: Glucocorticoid regulation of the inflammatory response to injury. Acta Anaesthesiol Scand 2004;48:799-813.

-6 Frank MG, Miguel ZD, Watkins LR, Maier SF: Prior exposure to glucocorticoids sensitizes the neuroinflammatory and peripheral inflammatory responses to E. coli lipopolysaccharide. Brain Behav Immun 2009;24:1930.

7 Smyth GP, Stapleton PP, Freeman TA, Concannon EM, Mestre JR, Duff M, Maddali S, Daly JM: Glucocorticoid pretreatment induces cytokine overexpression and nuclear factor-kappab activation in macrophages. J Surg Res 2004;116:253-261.

8 Viswanathan K, Dhabhar FS: Stress-induced enhancement of leukocyte trafficking into sites of surgery or immune activation. Proc Natl Acad Sci USA 2005;102:5808-5813.

$\checkmark 9$ Barber AE, Coyle SM, Marano MA, Fischer E, Calvano SE, Fong Y, Moldawer LL, Lowry SF: Glucocorticoid therapy alters hormonal and cytokine responses to endotoxin in man. J Immunol 1993;150:1999-2006.

10 Galon J, Franchimont D, Hiroi N, Frey G, Boettner A, Ehrhart-Bornstein M, O'Shea JJ, Chrousos GP, Bornstein SR: Gene profiling reveals unknown enhancing and suppressive actions of glucocorticoids on immune cells. FASEB J 2002;16:61-71.

11 Perry VH: Stress primes microglia to the presence of systemic inflammation: implications for environmental influences on the brain. Brain Behav Immun 2007;21:45-46.

12 Goujon E, Parnet P, Laye S, Combe C, Kelley KW, Dantzer R: Stress downregulates lipopolysaccharide-induced expression of proinflammatory cytokines in the spleen, pituitary, and brain of mice. Brain Behav Immun 1995; 9:292-303.

13 Dhabhar FS, McEwen BS: Acute stress enhances while chronic stress suppresses cellmediated immunity in vivo: a potential role for leukocyte trafficking. Brain Behav Immun 1997;11:286-306

14 Bowers SL, Bilbo SD, Dhabhar FS, Nelson RJ: Stressor-specific alterations in corticosterone and immune responses in mice. Brain Behav Immun 2008;22:105-113.

15 de Pablos RM, Villaran RF, Arguelles S, Herrera AJ, Venero JL, Ayala A, Cano J, Machado A: Stress increases vulnerability to inflammation in the rat prefrontal cortex. J Neurosci 2006;26:5709-5719.

16 Munhoz CD, Lepsch LB, Kawamoto EM, Malta MB, Lima Lde S, Avellar MC, Sapolsky RM, Scavone C: Chronic unpredictable stress exacerbates lipopolysaccharide-induced activation of nuclear factor-kappab in the frontal cortex and hippocampus via glucocorticoid secretion. J Neurosci 2006;26:3813-3820.

17 Dinkel K, MacPherson A, Sapolsky RM: Novel glucocorticoid effects on acute inflammation in the CNS. J Neurochem 2003;84:705716.

18 Sapolsky RM: Stress, glucocorticoids, and damage to the nervous system: the current state of confusion. Stress 1996;1:1-19.

19 Stein-Behrens BA, Elliott EM, Miller CA, Schilling JW, Newcombe R, Sapolsky RM: Glucocorticoids exacerbate kainic acid-induced extracellular accumulation of excitatory amino acids in the rat hippocampus. J Neurochem 1992;58:1730-1735.

20 Elliott EM, Sapolsky RM: Corticosterone impairs hippocampal neuronal calcium regulation - possible mediating mechanisms. Brain Res 1993;602:84-90.

21 McIntosh LJ, Sapolsky RM: Glucocorticoids increase the accumulation of reactive oxygen species and enhance adriamycin-induced toxicity in neuronal culture. Exp Neurol 1996; 141:201-206.

22 De Bosscher K, Vanden Berghe W, Haegeman G: The interplay between the glucocorti- coid receptor and nuclear factor-kappab or activator protein-1: molecular mechanisms for gene repression. Endocr Rev 2003;24:488 522

23 Goujon E, Parnet P, Cremona S, Dantzer R Endogenous glucocorticoids down regulate central effects of interleukin-1 beta on body temperature and behaviour in mice. Brain Res 1995;702:173-180.

24 Uz T, Dwivedi Y, Savani PD, Impagnatiello F, Pandey G, Manev H: Glucocorticoids stimulate inflammatory 5-lipoxygenase gene expression and protein translocation in the brain. J Neurochem 1999;73:693-699.

25 Hara H, Friedlander RM, Gagliardini V, Ayata C, Fink K, Huang Z, Shimizu-Sasamata M, Yuan J, Moskowitz MA: Inhibition of interleukin 1 beta converting enzyme family proteases reduces ischemic and excitotoxic neuronal damage. Proc Natl Acad Sci USA 1997;94: 2007-2012.

-26 Kawaguchi K, Hickey RW, Rose ME, Zhu L, Chen J, Graham SH: Cyclooxygenase-2 expression is induced in rat brain after kainateinduced seizures and promotes neuronal death in CA3 hippocampus. Brain Res 2005; 1050:130-137.

27 Takemiya T, Maehara M, Matsumura K, Yasuda S, Sugiura H, Yamagata K: Prostaglandin $\mathrm{E}_{2}$ produced by late induced COX-2 stimulates hippocampal neuron loss after seizure in the CA3 region. Neurosci Res 2006;56:103110

28 Cho IH, Hong J, Suh EC, Kim JH, Lee H, Lee JE, Lee S, Kim CH, Kim DW, Jo EK, Lee KE, Karin M, Lee SJ: Role of microglial IKKbeta in kainic acid-induced hippocampal neuronal cell death. Brain 2008;131:3019-3033.

-29 Monje ML, Toda H, Palmer TD: Inflammatory blockade restores adult hippocampal neurogenesis. Science 2003;302:17601765.

30 Yrjanheikki J, Tikka T, Keinanen R, Goldsteins G, Chan PH, Koistinaho J: A tetracycline derivative, minocycline, reduces inflammation and protects against focal cerebral ischemia with a wide therapeutic window. Proc Natl Acad Sci USA 199;96: 13496-13500.

31 Sapolsky R, Brooke S, Stein-Behrens B: Methodologic issues in studying glucocorticoidinduced damage to neurons. J Neurosci Methods 1995;58:1-15.
GCs Worsen CNS Inflammation and Excitotoxicity
Neuroendocrinology 2014;100:129-140 DOI: $10.1159 / 000367849$ 
32 MacPherson A, Dinkel K, Sapolsky R: Glucocorticoids worsen excitotoxin-induced expression of pro-inflammatory cytokines in hippocampal cultures. Exp Neurol 2005;194: 376-383.

33 Nimmerjahn A, Kirchhoff F, Helmchen F: Resting microglial cells are highly dynamic surveillants of brain parenchyma in vivo. Science 2005;308:1314-1318.

34 Waters JC: Accuracy and precision in quantitative fluorescence microscopy. J Cell Biol 2009;185:1135-1148.

35 Zhao S, Fernald RD: Comprehensive algorithm for quantitative real-time polymerase chain reaction. J Comput Biol 2005;12:10471064.

36 Rong Y, Baudry M: Seizure activity results in a rapid induction of nuclear factor-kappa $b$ in adult but not juvenile rat limbic structures. J Neurochem 1996;67:662-668.

-37 Wang DD, Englot DJ, Garcia PA, Lawton MT Young WL: Minocycline- and tetracyclineclass antibiotics are protective against partial seizures in vivo. Epilepsy Behav 2012;24:314318.

38 Kim HS, Suh YH: Minocycline and neurodegenerative diseases. Behav Brain Res 2009; 196:168-179.

39 Sapolsky RM, Pulsinelli WA: Glucocorticoids potentiate ischemic injury to neurons: therapeutic implications. Science 1985;229:13971400.
40 Biber K, Neumann H, Inoue K, Boddeke HW: Neuronal 'on' and 'off signals control microglia. Trends Neurosci 2007;30:596-602.

41 Hoek RM, Ruuls SR, Murphy CA, Wright GJ, Goddard R, Zurawski SM, Blom B, Homola ME, Streit WJ, Brown MH, Barclay AN, Sedgwick JD: Down-regulation of the macrophage lineage through interaction with OX2 (CD200). Science 2000;290:17681771.

42 Mott RT, Ait-Ghezala G, Town T, Mori T, Vendrame M, Zeng J, Ehrhart J, Mullan M, Tan J: Neuronal expression of CD22: novel mechanism for inhibiting microglial proinflammatory cytokine production. Glia 2004; 46:369-379.

43 Ransohoff RM, Cardona AE: The myeloid cells of the central nervous system parenchyma. Nature 2010;468:253-262.

44 Sapolsky RM, Romero LM, Munck AU: How do glucocorticoids influence stress responses? Integrating permissive, suppressive, stimulatory, and preparative actions. Endocr Rev 2000;21:55-89.

45 Cardona AE, Pioro EP, Sasse ME, Kostenko V, Cardona SM, Dijkstra IM, Huang D, Kidd G, Dombrowski S, Dutta R, Lee JC, Cook DN, Jung S, Lira SA, Littman DR, Ransohoff RM: Control of microglial neurotoxicity by the fractalkine receptor. Nat Neurosci 2006;9: 917-924.
46 Nair A, Bonneau RH: Stress-induced elevation of glucocorticoids increases microglia proliferation through NMDA receptor activation. J Neuroimmunol 2006;171:72-85.

47 Harrison JK, Jiang Y, Chen S, Xia Y, Maciejewski D, McNamara RK, Streit WJ, Salafranca MN, Adhikari S, Thompson DA, Botti P, Bacon KB, Feng L: Role for neuronally derived fractalkine in mediating interactions between neurons and CX3CR1-expressing microglia. Proc Natl Acad Sci USA 1998;95: 10896-10901.

48 Bhavsar PK, Sukkar MB, Khorasani N, Lee KY, Chung KF: Glucocorticoid suppression of CX3CL1 (fractalkine) by reduced gene promoter recruitment of NF-kappaB. FASEB J 2008;22:1807-1816.

49 Wiegers GJ, Reul JM: Induction of cytokine receptors by glucocorticoids: functional and pathological significance. Trends Pharmacol Sci 1998;19:317-321.

50 Roy M, Sapolsky RM: The exacerbation of hippocampal excitotoxicity by glucocorticoids is not mediated by apoptosis. Neuroendocrinology 2003;77:24-31.

51 Yeager MP, Rassias AJ, Pioli PA, Beach ML, Wardwell K, Collins JE, Lee HK, Guyre PM: Pretreatment with stress cortisol enhances the human systemic inflammatory response to bacterial endotoxin. Crit Care Med 2009;37: 2727-2732. 